\title{
Validation of the Adapted Response to Stressful Experiences Scale (RSES-4) Among First Responders
}

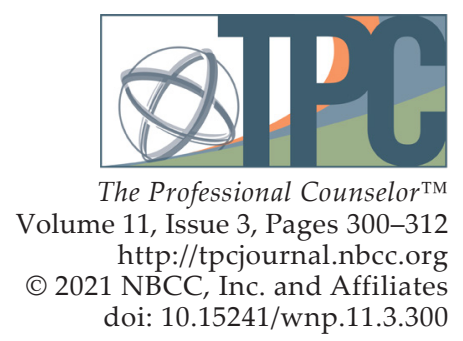

Warren N. Ponder, Elizabeth A. Prosek, Tempa Sherrill

First responders are continually exposed to trauma-related events. Resilience is evidenced as a protective factor for mental health among first responders. However, there is a lack of assessments that measure the construct of resilience from a strength-based perspective. The present study used archival data from a treatment-seeking sample of 238 first responders to validate the 22-item Response to Stressful Experiences Scale (RSES-22) and its abbreviated version, the RSES-4, with two confirmatory factor analyses. Using a subsample of 190 first responders, correlational analyses were conducted of the RSES-22 and RSES-4 with measures of depressive symptoms, post-traumatic stress, anxiety, and suicidality confirming convergent and criterion validity. The two confirmatory analyses revealed a poor model fit for the RSES-22; however, the RSES-4 demonstrated an acceptable model fit. Overall, the RSES-4 may be a reliable and valid measure of resilience for treatmentseeking first responder populations.

Keywords: first responders, resilience, assessment, mental health, confirmatory factor analysis

First responder populations (i.e., law enforcement, emergency medical technicians, and fire rescue) are often repeatedly exposed to traumatic and life-threatening conditions (Greinacher et al., 2019). Researchers have concluded that such critical incidents could have a deleterious impact on first responders' mental health, including the development of symptoms associated with post-traumatic stress, anxiety, depression, or other diagnosable mental health disorders (Donnelly \& Bennett, 2014; Jetelina et al., 2020; Klimley et al., 2018; Weiss et al., 2010). In a systematic review, Wild et al. (2020) suggested the promise of resilience-based interventions to relieve trauma-related psychological disorders among first responders. However, they noted the operationalization and measure of resilience as limitations to their intervention research. Indeed, researchers have conflicting viewpoints on how to define and assess resilience. For example, White et al. (2010) purported popular measures of resilience rely on a deficit-based approach. Counselors operate from a strength-based lens (American Counseling Association [ACA], 2014) and may prefer measures with a similar perspective. Additionally, counselors are mandated to administer assessments with acceptable psychometric properties that are normed on populations representative of the client (ACA, 2014, E.6.a., E.7.d.). For counselors working with first responder populations, resilience may be a factor of importance; however, appropriately measuring the construct warrants exploration. Therefore, the focus of this study was to validate a measure of resilience with strength-based principles among a sample of first responders.

\section{Risk and Resilience Among First Responders}

In a systematic review of the literature, Greinacher et al. (2019) described the incidents that first responders may experience as traumatic, including first-hand life-threatening events; secondary exposure and interaction with survivors of trauma; and frequent exposure to death, dead bodies,

Warren N. Ponder, PhD, is Director of Outcomes and Evaluation at One Tribe Foundation. Elizabeth A. Prosek, PhD, NCC, LPC, is an associate professor at Penn State University. Tempa Sherrill, MS, LPC-S, is the founder of Stay the Course and a volunteer at One Tribe Foundation. Correspondence may be addressed to Warren N. Ponder, 855 Texas St., Suite 105, Fort Worth, TX 76102 , warren@1tribefoundation.org. 
and injury. Law enforcement officers (LEOs) reported that the most severe critical incidents they encounter are making a mistake that injures or kills a colleague; having a colleague intentionally killed; and making a mistake that injures or kills a bystander (Weiss et al., 2010). Among emergency medical technicians (EMTs), critical incidents that evoked the most self-reported stress included responding to a scene involving family, friends, or others to the crew and seeing someone dying (Donnelly \& Bennett, 2014). Exposure to these critical incidents may have consequences for first responders. For example, researchers concluded first responders may experience mental health symptoms as a result of the stress-related, repeated exposure (Jetelina et al., 2020; Klimley et al., 2018; Weiss et al., 2010). Moreover, considering the cumulative nature of exposure (Donnelly \& Bennett, 2014), researchers concluded first responders are at increased risk for post-traumatic stress disorder (PTSD), depression, and generalized anxiety symptoms (Jetelina et al., 2020; Klimley et al., 2018; Weiss et al., 2010). Symptoms commonly experienced among first responders include those associated with post-traumatic stress, anxiety, and depression.

In a collective review of first responders, Kleim and Westphal (2011) determined a prevalence rate for PTSD of $8 \%-32 \%$, which is higher than the general population lifetime rate of $6.8-7.8 \%$ (American Psychiatric Association [APA], 2013; National Institute of Mental Health [NIMH], 2017). Some researchers have explored rates of PTSD by specific first responder population. For example, Klimley et al. (2018) concluded that $7 \%-19 \%$ of LEOs and $17 \%-22 \%$ of firefighters experience PTSD. Similarly, in a sample of LEOs, Jetelina and colleagues (2020) reported $20 \%$ of their participants met criteria for PTSD.

Generalized anxiety and depression are also prevalent mental health symptoms for first responders. Among a sample of firefighters and EMTs, $28 \%$ disclosed anxiety at moderate-severe and several levels (Jones et al., 2018). Furthermore, 17\% of patrol LEOs reported an overall prevalence of generalized anxiety disorder (Jetelina et al., 2020). Additionally, first responders may be at higher risk for depression (Klimley et al., 2018), with estimated prevalence rates of 16\%-26\% (Kleim \& Westphal, 2011). Comparatively, the past 12 -month rate of major depressive disorder among the general population is $7 \%$ (APA, 2013). In a recent study, $16 \%$ of LEOs met criteria for major depressive disorder (Jetelina et al., 2020). Moreover, in a sample of firefighters and EMTs, $14 \%$ reported moderate-severe and severe depressive symptoms (Jones et al., 2018). Given these higher rates of distressful mental health symptoms, including post-traumatic stress, generalized anxiety, and depression, protective factors to reduce negative impacts are warranted.

\section{Resilience}

Broadly defined, resilience is "the ability to adopt to and rebound from change (whether it is from stress or adversity) in a healthy, positive and growth-oriented manner" (Burnett, 2017, p. 2). White and colleagues (2010) promoted a positive psychology approach to researching resilience, relying on strength-based characteristics of individuals who adapt after a stressor event. Similarly, other researchers explored how individuals' cognitive flexibility, meaning-making, and restoration offer protection that may be collectively defined as resilience (Johnson et al., 2011).

A key element among definitions of resilience is one's exposure to stress. Given their exposure to trauma-related incidents, first responders require the ability to cope or adapt in stressful situations (Greinacher et al., 2019). Some researchers have defined resilience as a strength-based response to stressful events (Burnett, 2017), in which healthy coping behaviors and cognitions allow individuals to overcome adverse experiences (Johnson et al., 2011; White et al., 2010). When surveyed about positive coping strategies, first responders most frequently reported resilience as important to their well-being (Crowe et al., 2017). 
Researchers corroborated the potential impact of resilience for the population. For example, in samples of LEOs, researchers confirmed resilience served as a protective factor for PTSD (Klimley et al., 2018) and as a mediator between social support and PTSD symptoms (McCanlies et al., 2017). In a sample of firefighters, individual resilience mediated the indirect path between traumatic events and global perceived stress of PTSD, along with the direct path between traumatic events and PTSD symptoms (Lee et al., 2014). Their model demonstrated that those with higher levels of resilience were more protected from traumatic stress. Similarly, among emergency dispatchers, resilience was positively correlated with positive affect and post-traumatic growth, and negatively correlated with job stress (Steinkopf et al., 2018). The replete associations of resilience as a protective factor led researchers to develop resilience-based interventions. For example, researchers surmised promising results from mindfulness-based resilience interventions for firefighters (Joyce et al., 2019) and LEOs (Christopher et al., 2018). Moreover, Antony and colleagues (2020) concluded that resilience training programs demonstrated potential to reduce occupational stress among first responders.

\section{Assessment of Resilience}

Recognizing the significance of resilience as a mediating factor in PTSD among first responders and as a promising basis for interventions when working with LEOs, a reliable means to measure it among first responder clients is warranted. In a methodological review of resilience assessments, Windle and colleagues (2011) identified 19 different measures of resilience. They found 15 assessments were from original development and validation studies with four subsequent validation manuscripts from their original assessment, of which none were developed with military or first responder samples.

Subsequently, Johnson et al. (2011) developed the Response to Stressful Experiences Scale (RSES-22) to assess resilience among military populations. Unlike deficit-based assessments of resilience, they proposed a multidimensional construct representing how individuals respond to stressful experiences in adaptive or healthy ways. Cognitive flexibility, meaning-making, and restoration were identified as key elements when assessing for individuals' characteristics connected to resilience when overcoming hardships. Initially they validated a five-factor structure for the RSES-22 with military active-duty and reserve components. Later, De La Rosa et al. (2016) re-examined the RSES-22. De La Rosa and colleagues discovered a unidimensional factor structure of the RSES-22 and validated a shorter 4-item subset of the instrument, the RSES-4, again among military populations.

It is currently unknown if the performance of the RSES-4 can be generalized to first responder populations. While there are some overlapping experiences between military populations and first responders in terms of exposure to trauma and high-risk occupations, the Substance Abuse and Mental Health Services Administration (SAMHSA; 2018) suggested differences in training and types of risk. In the counseling profession, these populations are categorized together, as evidenced by the Military and Government Counseling Association ACA division. Additionally, there may also be dual identities within the populations. For example, Lewis and Pathak (2014) found that $22 \%$ of LEOs and $15 \%$ of firefighters identified as veterans. Although the similarities of the populations may be enough to theorize the use of the same resilience measure, validation of the RSES-22 and RSES-4 among first responders remains unexamined.

\section{Purpose of the Study}

First responders are repeatedly exposed to traumatic and stressful events (Greinacher et al., 2019) and this exposure may impact their mental health, including symptoms of post-traumatic stress, anxiety, depression, and suicidality (Jetelina et al., 2020; Klimley et al., 2018). Though most measures of resilience are grounded in a deficit-based approach, researchers using a strength-based approach 
proposed resilience may be a protective factor for this population (Crowe et al., 2017; Wild et al., 2020). Consequently, counselors need a means to assess resilience in their clinical practice from a strength-based conceptualization of clients.

Johnson et al. (2011) offered a non-deficit approach to measuring resilience in response to stressful events associated with military service. Thus far, researchers have conducted analyses of the RSES-22 and RSES-4 with military populations (De La Rosa et al., 2016; Johnson et al., 2011; Prosek \& Ponder, 2021), but not yet with first responders. While there are some overlapping characteristics between the populations, there are also unique differences that warrant research with discrete sampling (SAMHSA, 2018). In light of the importance of resilience as a protective factor for mental health among first responders, the purpose of the current study was to confirm the reliability and validity of the RSES-22 and RSES-4 when utilized with this population. In the current study, we hypothesized the measures would perform similarly among first responders and if so, the RSES-4 would offer counselors a brief assessment option in clinical practice that is both reliable and valid.

\section{Method}

\section{Participants}

Participants in the current non-probability, purposive sample study were first responders $(N=238)$ seeking clinical treatment at an outpatient, mental health nonprofit organization in the Southwestern United States. Participants' mean age was 37.53 years $(S D=10.66)$. The majority of participants identified as men $(75.2 \% ; n=179)$, with women representing $24.8 \%(n=59)$ of the sample. In terms of race and ethnicity, participants identified as White $(78.6 \% ; n=187)$, Latino/a $(11.8 \% ; n=28)$, African American or Black (5.5\%; $n=13)$, Native American $(1.7 \% ; n=4)$, Asian American $(1.3 \% ; n=3)$, and multiple ethnicities $(1.3 \% ; n=3)$. The participants identified as first responders in three main categories: LEO $(34.9 \% ; n=83)$, EMT $(28.2 \% ; n=67)$, and fire rescue $(25.2 \% ; n=60)$. Among the first responders, $26.9 \%$ reported previous military affiliation. As part of the secondary analysis, we utilized a subsample $(n=190)$ that was reflective of the larger sample (see Table 1).

\section{Procedure}

The data for this study were collected between 2015-2020 as part of the routine clinical assessment procedures at a nonprofit organization serving military service members, first responders, frontline health care workers, and their families. The agency representatives conduct clinical assessments with clients at intake, Session 6, Session 12, and Session 18 or when clinical services are concluded. We consulted with the second author's Institutional Review Board, which determined the research as exempt, given the de-identified, archival nature of the data. For inclusion in this analysis, data needed to represent first responders, ages 18 or older, with a completed RSES-22 at intake. The RSES- 4 are four questions within the RSES-22 measure; therefore, the participants did not have to complete an additional measure. For the secondary analysis, data from participants who also completed other mental health measures at intake were also included (see Measures). 


\section{Table 1}

Demographics of Sample

\begin{tabular}{lcc}
\hline \multicolumn{1}{c}{ Characteristic } & $\begin{array}{c}\text { Sample 1 } \\
(\boldsymbol{N}=\mathbf{2 3 8})\end{array}$ & $\begin{array}{c}\text { Sample 2 } \\
(\boldsymbol{n}=\mathbf{1 9 0})\end{array}$ \\
\hline Age (Years) & & \\
Mean & 37.53 & 37.12 \\
Median & 35.50 & 35.00 \\
SD & 10.66 & 10.30 \\
$\quad$ Range & 46 & 45 \\
Time in Service (Years) & & \\
$\quad$ Mean & 11.62 & 11.65 \\
Median & 10.00 & 10.00 \\
SD & 9.33 & 9.37 \\
Range & 41 & 39 \\
\hline & \multicolumn{2}{c}{$n \mathbf{n ( \% )}$} \\
\hline
\end{tabular}

$\begin{array}{lcc}\text { First Responder Type } & & \\ \text { Emergency Medical } & 67(28.2 \%) & 54(28.4 \%) \\ \text { Technicians } & 60(25.2 \%) & 45(23.7 \%) \\ \text { Fire Rescue } & 83(34.9 \%) & 72(37.9 \%) \\ \text { Law Enforcement } & 9(3.8 \%) & 5(2.6 \%) \\ \text { Other } & 10(4.2 \%) & 6(3.2 \%) \\ \text { Two or more } & 9(3.8 \%) & 8(4.2 \%) \\ \text { Not reported } & & \\ \text { Gender } & 59(24.8 \%) & 47(24.7 \%) \\ \text { Women } & 179(75.2 \%) & 143(75.3 \%) \\ \text { Men } & & \\ \text { Ethnicity } & 13(5.5 \%) & 8(4.2 \%) \\ \text { African American/Black } & 3(1.3 \%) & 3(1.6 \%) \\ \text { Asian American } & 28(11.8 \%) & 24(12.6 \%) \\ \text { Latino(a)/Hispanic } & 3(1.3 \%) & 3(1.6 \%) \\ \text { Multiple Ethnicities } & 4(1.7 \%) & 3(1.6 \%) \\ \text { Native American } & 187(78.6 \%) & 149(78.4 \%) \\ \text { White } & \end{array}$

Note. Sample 2 is a subset of Sample 1. Time in service for Sample 1, $n=225$; time in service for Sample 2, $n=190$.

\section{Measures}

\section{Response to Stressful Experiences Scale}

The Response to Stressful Experiences Scale (RSES-22) is a 22-item measure to assess dimensions of resilience, including meaning-making, active coping, cognitive flexibility, spirituality, and selfefficacy (Johnson et al., 2011). Participants respond to the prompt "During and after life's most 
stressful events, I tend to" on a 5-point Likert scale from 0 (not at all like me) to 4 (exactly like me). Total scores range from 0 to 88 in which higher scores represent greater resilience. Example items include see it as a challenge that will make me better, pray or meditate, and find strength in the meaning, purpose, or mission of my life. Johnson et al. (2011) reported the RSES-22 demonstrates good internal consistency $(\alpha=.92)$ and test-retest reliability $(\alpha=.87)$ among samples from military populations. Further, the developers confirmed convergent, discriminant, concurrent, and incremental criterion validity (see Johnson et al., 2011). In the current study, Cronbach's alpha of the total score was .93.

\section{Adapted Response to Stressful Experiences Scale}

The adapted Response to Stressful Experiences Scale (RSES-4) is a 4-item measure to assess resilience as a unidimensional construct (De La Rosa et al., 2016). The prompt and Likert scale are consistent with the original RSES-22; however, it only includes four items: find a way to do what's necessary to carry on, know I will bounce back, learn important and useful life lessons, and practice ways to handle it better next time. Total scores range from 0 to 16, with higher scores indicating greater resilience. De La Rosa et al. (2016) reported acceptable internal consistency $(\alpha=.76-.78)$, test-retest reliability, and demonstrated criterion validity among multiple military samples. In the current study, the Cronbach's alpha of the total score was .74.

\section{Patient Health Questionnaire-9}

The Patient Health Questionnaire-9 (PHQ-9) is a 9-item measure to assess depressive symptoms in the past 2 weeks (Kroenke et al., 2001). Respondents rate the frequency of their symptoms on a 4-point Likert scale ranging from 0 (not at all) to 3 (nearly every day). Total scores range from 0 to 27, in which higher scores indicate increased severity of depressive symptoms. Example items include little interest or pleasure in doing things and feeling tired or having little energy. Kroenke et al. (2001) reported good internal consistency $(\alpha=.89)$ and established criterion and construct validity. In this sample, Cronbach's alpha of the total score was .88.

\section{PTSD Checklist-5}

The PTSD Checklist-5 (PCL-5) is a 20-item measure for the presence of PTSD symptoms in the past month (Blevins et al., 2015). Participants respond on a 5-point Likert scale indicating frequency of PTSDrelated symptoms from 0 (not at all) to 4 (extremely). Total scores range from 0 to 80, in which higher scores indicate more severity of PTSD-related symptoms. Example items include repeated, disturbing dreams of the stressful experience and trouble remembering important parts of the stressful experience. Blevins et al. (2015) reported good internal consistency $(\alpha=.94)$ and determined convergent and discriminant validity. In this sample, Cronbach's alpha of the total score was .93.

\section{Generalized Anxiety Disorder-7}

The Generalized Anxiety Disorder-7 (GAD-7) is a 7-item measure to assess for anxiety symptoms over the past 2 weeks (Spitzer et al., 2006). Participants rate the frequency of the symptoms on a 4-point Likert scale ranging from 0 (not at all) to 3 (nearly every day). Total scores range from 0 to 21 with higher scores indicating greater severity of anxiety symptoms. Example items include not being able to stop or control worrying and becoming easily annoyed or irritable. Among patients from primary care settings, Spitzer et al. (2006) determined good internal consistency $(\alpha=.92)$ and established criterion, construct, and factorial validity. In this sample, Cronbach's alpha of the total score was .91.

\section{Suicidal Behaviors Questionnaire-Revised}

The Suicidal Behaviors Questionnaire-Revised (SBQ-R) is a 4-item measure to assess suicidality (Osman et al., 2001). Each item assesses a different dimension of suicidality: lifetime ideation and 
attempts, frequency of ideation in the past 12 months, threat of suicidal behaviors, and likelihood of suicidal behaviors (Gutierrez et al., 2001). Total scores range from 3 to 18, with higher scores indicating more risk of suicide. Example items include How often have you thought about killing yourself in the past year? and How likely is it that you will attempt suicide someday? In a clinical sample, Osman et al. (2001) reported good internal consistency $(\alpha=.87)$ and established criterion validity. In this sample, Cronbach's alpha of the total score was .85 .

\section{Data Analysis}

Statistical analyses were conducted using SPSS version 26.0 and SPSS Analysis of Moment Structures (AMOS) version 26.0. We examined the dataset for missing values, replacing $0.25 \%$ ( 32 of 12,836 values) of data with series means. We reviewed descriptive statistics of the RSES-22 and RSES-4 scales. We determined multivariate normality as evidenced by skewness less than 2.0 and kurtosis less than 7.0 (Dimitrov, 2012). We assessed reliability for the scales by interpreting Cronbach's alphas and inter-item correlations to confirm internal consistency.

We conducted two separate confirmatory factor analyses to determine the model fit and factorial validity of the 22-item measure and adapted 4-item measure. We used several indices to conclude model fit: minimum discrepancy per degree of freedom (CMIN/DF) and $p$-values, root mean residual (RMR), goodness-of-fit index (GFI), comparative fit index (CFI), Tucker-Lewis index (TLI), and the root mean square error of approximation (RMSEA). According to Dimitrov (2012), values for the CMIN/DF < 2.0, $p>.05$, RMR $<.08$, GFI $>.90$, CFI $>.90$, TLI $>.90$, and RMSEA $<.10$ provide evidence of a strong model fit. To determine criterion validity, we assessed a subsample of participants $(n=190)$ who had completed the RSES-22, RSES-4, and four other psychological measures (i.e., PHQ-9, PCL-5, GAD-7, and SBQ-R). We determined convergent validity by conducting bivariate correlations between the RSES-22 and RSES- 4 .

\section{Results}

\section{Descriptive Analyses}

We computed means, standard deviations, 95\% confidence interval (CI), and score ranges for the RSES-22 and RSES-4 (Table 2). Scores on the RSES-22 ranged from 19-88. Scores on the RSES-4 ranged from 3-16. Previous researchers using the RSES-22 on military samples reported mean scores of 57.64-70.74 with standard deviations between 8.15-15.42 (Johnson et al., 2011; Prosek \& Ponder, 2021). In previous research of the RSES-4 with military samples, mean scores were 9.95-11.20 with standard deviations between 3.02-3.53 (De La Rosa et al., 2016; Prosek \& Ponder, 2021).

\section{Table 2}

Descriptive Statistics for RSES-22 and RSES-4

\begin{tabular}{ccccc}
\hline Variable & $\boldsymbol{M}$ & $\mathrm{SD}$ & $\mathbf{9 5 \%} \mathbf{C I}$ & Score Range \\
\hline RSES-22 scores & 60.12 & 13.76 & $58.52,61.86$ & $19-88$ \\
RSES-4 scores & 11.66 & 2.62 & $11.33,11.99$ & $3-16$ \\
\hline
\end{tabular}

Note. $N=238$. RSES-22 = Response to Stressful Experiences Scale 22-item; RSES-4 = Response to Stressful Experiences Scale 4-item adaptation. 


\section{Reliability Analyses}

To determine the internal consistency of the resiliency measures, we computed Cronbach's alphas. For the RSES-22, we found strong evidence of inter-item reliability $(\alpha=.93)$, which was consistent with the developers' estimates $(\alpha=.93$; Johnson et al., 2011). For the RSES-4, we assessed acceptable inter-item reliability $(\alpha=.74)$, which was slightly lower than previous estimates $(\alpha=.76-.78$; De La Rosa et al., 2016). We calculated the correlation between items and computed the average of all the coefficients. The average inter-item correlation for the RSES-22 was .38, which falls within the acceptable range (.15-.50). The average inter-item correlation for the RSES-4 was .51, slightly above the acceptable range. Overall, evidence of internal consistency was confirmed for each scale.

\section{Factorial Validity Analyses}

We conducted two confirmatory factor analyses to assess the factor structure of the RSES-22 and RSES-4 for our sample of first responders receiving mental health services at a community clinic (Table 3). For the RSES-22, a proper solution converged in 10 iterations. Item loadings ranged between $.31-.79$, with 15 of 22 items loading significantly (> .6) on the latent variable. It did not meet statistical criteria for good model fit: $\chi^{2}(209)=825.17, p=.000,90 \%$ CI [0.104, 0.120]. For the RSES-4, a proper solution converged in eight iterations. Item loadings ranged between .47-.80, with three of four items loading significantly ( $>.6)$ on the latent variable. It met statistical criteria for good model fit: $\chi^{2}(2)=5.89, p=.053,90 \%$ CI [0.000, 0.179]. The CMIN/DF was above the suggested < 2.0 benchmark; however, the other fit indices indicated a model fit.

\section{Table 3}

Confirmatory Factor Analysis Fit Indices for RSES-22 and RSES-4

\begin{tabular}{lrrrrrrrrr}
\hline \multicolumn{1}{c}{ Variable } & \multicolumn{1}{c}{$d f$} & \multicolumn{1}{c}{$\chi^{2}$} & $\begin{array}{c}\text { CMIN/ } \\
\text { DF }\end{array}$ & RMR & GFI & CFI & TLI & RMSEA & 90\% CI \\
\hline RSES-22 & 209 & $825.17 / .000$ & 3.95 & .093 & .749 & .771 & 0.747 & .112 & $0.104,0.120$ \\
RSES-4 & 2 & $5.89 / .053$ & 2.94 & .020 & .988 & .981 & 0.944 & .091 & $0.000,0.179$ \\
\hline
\end{tabular}

Note. $N=$ 238. RSES-22 = Response to Stressful Experiences Scale 22-item; RSES-4 = Response to Stressful Experiences Scale 4-item adaptation; CMIN/DF = Minimum Discrepancy per Degree of Freedom; RMR = Root Mean Square Residual; GFI = Goodness-of-Fit Index; CFI = Comparative Fit Index; TLI = Tucker-Lewis Index; RMSEA = Root Mean Squared Error of Approximation.

\section{Criterion and Convergent Validity Analyses}

To assess for criterion validity of the RSES-22 and RSES-4, we conducted correlational analyses with four established psychological measures (Table 4). We utilized a subsample of participants $(n=190)$ who completed the PHQ-9, PCL-5, GAD-7, and SBQ-R at intake. Normality of the data was not a concern because analyses established appropriate ranges for skewness and kurtosis $( \pm 1.0)$. The internal consistency of the RSES-22 $(\alpha=.93)$ and RSES-4 $(\alpha=.77)$ of the subsample was comparable to the larger sample and previous studies. The RSES-22 and RSES-4 related to the psychological measures of distress in the expected direction, meaning measures were significantly and negatively related, indicating that higher resiliency scores were associated with lower scores of symptoms associated with diagnosable mental health disorders (i.e., post-traumatic stress, anxiety, depression, 
and suicidal behavior). We verified convergent validity with a correlational analysis of the RSES-22 and RSES-4, which demonstrated a significant and positive relationship.

\section{Table 4}

Criterion and Convergent Validity of RSES-22 and RSES-4

\begin{tabular}{lccccccc}
\hline & $M(S D)$ & Cronbach's $\alpha$ & RSES-22 & PHQ-9 & PCL-5 & GAD-7 & SBQ-R \\
\hline RSES-22 & $60.16(14.17)$ & .93 & -- & $-.287^{*}$ & $-.331^{*}$ & $-.215^{*}$ & $-.346^{*}$ \\
RSES-4 & $11.65(2.68)$ & .77 & .918 & $-.290^{*}$ & $-.345^{*}$ & $-.220^{*}$ & $-.327^{*}$ \\
\hline
\end{tabular}

Note. $n=190$. RSES-22 $=$ Response to Stressful Experiences Scale 22-item; RSES-4 $=$ Response to Stressful Experiences Scale 4-item adaptation; PHQ-9 = Patient Health Questionnaire-9; PCL-5 = PTSD Checklist-5; GAD-7 = Generalized Anxiety Disorder-7; SBQ-R = Suicidal Behaviors Questionnaire-Revised. ${ }^{*} p<.01$.

\section{Discussion}

The purpose of this study was to validate the factor structure of the RSES-22 and the abbreviated RSES-4 with a first responder sample. Aggregated means were similar to those in the articles that validated and normed the measures in military samples (De La Rosa et al., 2016; Johnson et al., 2011; Prosek \& Ponder, 2021). Additionally, the internal consistency was similar to previous studies. In the original article, Johnson et al. (2011) proposed a five-factor structure for the RSES-22, which was later established as a unidimensional assessment after further exploratory factor analysis (De La Rosa et al., 2016). Subsequently, confirmatory factor analyses with a treatment-seeking veteran population revealed that the RSES-22 demonstrated unacceptable model fit, whereas the RSES-4 demonstrated a good model fit (Prosek \& Ponder, 2021). In both samples, the RSES-4 GFI, CFI, and TLI were all .944 or higher, whereas the RSES-22 GFI, CFI, and TLI were all .771 or lower. Additionally, criterion and convergent validity as measured by the PHQ-9, PCL-5, and GAD-7 in both samples were extremely close. Similarly, in this sample of treatment-seeking first responders, confirmatory factor analyses indicated an inadequate model fit for the RSES-22 and a good model fit for the RSES-4. Lastly, convergent and criterion validity were established with correlation analyses of the RSES-22 and RSES-4 with four other standardized assessment instruments (i.e., PHQ-9, PCL-5, GAD-7, SBQ-R). We concluded that among the first responder sample, the RSES- 4 demonstrated acceptable psychometric properties, as well as criterion and convergent validity with other mental health variables (i.e., posttraumatic stress, anxiety, depression, and suicidal behavior).

\section{Implications for Clinical Practice}

First responders are a unique population and are regularly exposed to trauma (Donnelly \& Bennett, 2014; Jetelina et al., 2020; Klimley et al., 2018; Weiss et al., 2010). Although first responders could potentially benefit from espousing resilience, they are often hesitant to seek mental health services (Crowe et al., 2017; Jones, 2017). The RSES-22 and RSES-4 were originally normed with military populations. The results of the current study indicated initial validity and reliability among a first responder population, revealing that the RSES-4 could be useful for counselors in assessing resilience. 
It is important to recognize that first responders have perceived coping with traumatic stress as an individual process (Crowe et al., 2017) and may believe that seeking mental health services is counter to the emotional and physical training expectations of the profession (Crowe et al., 2015). Therefore, when first responders seek mental health care, counselors need to be prepared to provide culturally responsive services, including population-specific assessment practices and resilience-oriented care.

Jones (2017) encouraged a comprehensive intake interview and battery of appropriate assessments be conducted with first responder clients. Counselors need to balance the number of intake questions while responsibly assessing for mental health comorbidities such as post-traumatic stress, anxiety, depression, and suicidality. The RSES-4 provides counselors a brief, yet targeted assessment of resilience.

Part of what cultural competency entails is assessing constructs (e.g., resilience) that have been shown to be a protective factor against PTSD among first responders (Klimley et al., 2018). Since the items forming the RSES-4 were developed to highlight the positive characteristics of coping (Johnson et al., 2011), rather than a deficit approach, this aligns with the grounding of the counseling profession. It is also congruent with first responders' perceptions of resilience. Indeed, in a content analysis of focus group interviews with first responders, participants defined resilience as a positive coping strategy that involves emotional regulation, perseverance, personal competence, and physical fitness (Crowe et al., 2017).

The RSES-4 is a brief, reliable, and valid measure of resilience with initial empirical support among a treatment-seeking first responder sample. In accordance with the ACA (2014) Code of Ethics, counselors are to administer assessments normed with the client population (E.8.). Thus, the results of the current study support counselors' use of the measure in practice. First responder communities are facing unprecedented work tasks in response to COVID-19. Subsequently, their mental health might suffer (Centers for Disease Control and Prevention, 2020) and experts have recommended promoting resilience as a protective factor for combating the negative mental health consequences of COVID-19 (Chen \& Bonanno, 2020). Therefore, the relevance of assessing resilience among first responder clients in the current context is evident.

\section{Limitations and Future Research}

This study is not without limitations. The sample of first responders was homogeneous in terms of race, ethnicity, and gender. Subsamples of first responders (i.e., LEO, EMT, fire rescue) were too small to conduct within-group analyses to determine if the factor structure of the RSES-22 and RSES-4 would perform similarly. Also, our sample of first responders included two emergency dispatchers. Researchers reported that emergency dispatchers should not be overlooked, given an estimated 13\% to $15 \%$ of emergency dispatchers experience post-traumatic symptomatology (Steinkopf et al., 2018). Future researchers may develop studies that further explore how, if at all, emergency dispatchers are represented in first responder research.

Furthermore, future researchers could account for first responders who have prior military service. In a study of LEOs, Jetelina et al. (2020) found that participants with military experience were 3.76 times more likely to report mental health concerns compared to LEOs without prior military affiliation. Although we reported the prevalence rate of prior military experience in our sample, the within-group sample size was not sufficient for additional analyses. Finally, our sample represented treatment-seeking first responders. Future researchers may replicate this study with non-treatmentseeking first responder populations. 


\section{Conclusion}

First responders are at risk for sustaining injuries, experiencing life-threatening events, and witnessing harm to others (Lanza et al., 2018). The nature of their exposure can be repeated and cumulative over time (Donnelly \& Bennett, 2014), indicating an increased risk for post-traumatic stress, anxiety, and depressive symptoms, as well as suicidal behavior (Jones et al., 2018). Resilience is a promising protective factor that promotes wellness and healthy coping among first responders (Wild et al., 2020), and counselors may choose to routinely measure for resilience among first responder clients. The current investigation concluded that among a sample of treatment-seeking first responders, the original factor structure of the RSES-22 was unstable, although it demonstrated good reliability and validity. The adapted version, RSES-4, demonstrated good factor structure while also maintaining acceptable reliability and validity, consistent with studies of military populations (De La Rosa et al., 2016; Johnson et al., 2011; Prosek \& Ponder, 2021). The RSES-4 provides counselors with a brief and strength-oriented option for measuring resilience with first responder clients.

\section{Conflict of Interest and Funding Disclosure}

The authors reported no conflict of interest or funding contributions for the development of this manuscript.

\section{References}

American Counseling Association. (2014). ACA code of ethics.

American Psychiatric Association. (2013). Diagnostic and statistical manual of mental disorders (5th ed.).

Antony, J., Brar, R., Khan, P. A., Ghassemi, M., Nincic, V., Sharpe, J. P., Straus, S. E., \& Tricco, A. C. (2020). Interventions for the prevention and management of occupational stress injury in first responders: A rapid overview of reviews. Systematic Reviews, 9(121), 1-20. https://doi.org/10.1186/s13643-020-01367-w

Blevins, C. A., Weathers, F. W., Davis, M. T., Witte, T. K., \& Domino, J. L. (2015). The Posttraumatic Stress Disorder Checklist for DSM-5 (PCL-5): Development and initial psychometric evaluation. Journal of Traumatic Stress, 28(6), 489-498. https://doi.org/10.1002/jts.22059

Burnett, H. J., Jr. (2017). Revisiting the compassion fatigue, burnout, compassion satisfaction, and resilience connection among CISM responders. Journal of Police Emergency Response, 7(3), 1-10. https://doi.org/10.1177/2158244017730857

Centers for Disease Control and Prevention. (2020, June 30). Coping with stress. https://www.cdc.gov/cor onavirus/2019-ncov/daily-life-coping/managing-stress-anxiety.html

Chen, S., \& Bonanno, G. A. (2020). Psychological adjustment during the global outbreak of COVID-19: A resilience perspective. Psychological Trauma: Theory, Research, Practice, and Policy, 12(S1), S51-S54. https://doi.org/10.1037/tra0000685

Christopher, M. S., Hunsinger, M., Goerling, R. J., Bowen, S., Rogers, B. S., Gross, C. R., Dapolonia, E., \& Pruessner, J. C. (2018). Mindfulness-based resilience training to reduce health risk, stress reactivity, and aggression among law enforcement officers: A feasibility and preliminary efficacy trial. Psychiatry Research, 264, 104-115. https://doi.org/10.1016/j.psychres.2018.03.059

Crowe, A., Glass, J. S., Lancaster, M. F., Raines, J. M., \& Waggy, M. R. (2015). Mental illness stigma among first responders and the general population. Journal of Military and Government Counseling, 3(3), 132-149. http://mgcaonline.org/wp-content/uploads/2013/02/JMGC-Vol-3-Is-3.pdf

Crowe, A., Glass, J. S., Lancaster, M. F., Raines, J. M., \& Waggy, M. R. (2017). A content analysis of psychological resilience among first responders. SAGE Open, 7(1), 1-9. https://doi.org/10.1177/2158244017698530 
De La Rosa, G. M., Webb-Murphy, J. A., \& Johnston, S. L. (2016). Development and validation of a brief measure of psychological resilience: An adaptation of the Response to Stressful Experiences Scale. Military Medicine, 181(3), 202-208. https://doi.org/10.7205/MILMED-D-15-00037

Dimitrov, D. M. (2012). Statistical methods for validation of assessment scale data in counseling and related fields. American Counseling Association.

Donnelly, E. A., \& Bennett, M. (2014). Development of a critical incident stress inventory for the emergency medical services. Traumatology, 20(1), 1-8. https://doi.org/10.1177/1534765613496646

Greinacher, A., Derezza-Greeven, C., Herzog, W., \& Nikendei, C. (2019). Secondary traumatization in first responders: A systematic review. European Journal of Psychotraumatology, 10(1), 1562840. https://doi.org/10.1080/20008198.2018.1562840

Gutierrez, P. M., Osman, A., Barrios, F. X., \& Kopper, B. A. (2001). Development and initial validation of the Self-Harm Behavior Questionnaire. Journal of Personality Assessment, 77(3), 475-490. https://doi.org/10.1207/S15327752JPA7703_08

Jetelina, K. K., Mosberry, R. J., Gonzalez, J. R., Beauchamp, A. M., \& Hall, T. (2020). Prevalence of mental illnesses and mental health care use among police officers. JAMA Network Open, 3(10), 1-12. https://doi.org/10.1001/jamanetworkopen.2020.19658

Johnson, D. C., Polusny, M. A., Erbes, C. R., King, D., King, L., Litz, B. T., Schnurr, P. P., Friedman, M., Pietrzak, R. H., \& Southwick, S. M. (2011). Development and initial validation of the Response to Stressful Experiences Scale. Military Medicine, 176(2), 161-169. https://doi.org/10.7205/milmed-d-10-00258

Jones, S. (2017). Describing the mental health profile of first responders: A systematic review. Journal of the American Psychiatric Nurses Association, 23(3), 200-214. https://doi.org/10.1177/1078390317695266

Jones, S., Nagel, C., McSweeney, J., \& Curran, G. (2018). Prevalence and correlates of psychiatric symptoms among first responders in a Southern state. Archives of Psychiatric Nursing, 32(6), 828-835. https://doi.org/10.1016/j.apnu.2018.06.007

Joyce, S., Tan, L., Shand, F., Bryant, R. A., \& Harvey, S. B. (2019). Can resilience be measured and used to predict mental health symptomology among first responders exposed to repeated trauma? Journal of Occupational and Environmental Medicine, 61(4), 285-292. https://doi.org/10.1097/JOM.0000000000001526

Kleim, B., \& Westphal, M. (2011). Mental health in first responders: A review and recommendation for prevention and intervention strategies. Traumatology, 17(4), 17-24. https://doi.org/10.1177/1534765611429079

Klimley, K. E., Van Hasselt, V. B., \& Stripling, A. M. (2018). Posttraumatic stress disorder in police, firefighters, and emergency dispatchers. Aggression and Violent Behavior, 43, 33-44.

https://doi.org/10.1016/j.avb.2018.08.005

Kroenke, K., Spitzer, R. L., \& Williams, J. B. W. (2001). The PHQ-9: Validity of a brief depression severity measure. Journal of General Internal Medicine, 16, 606-613. https://doi.org/10.1046/j.1525-1497.2001.016009606.x

Lanza, A., Roysircar, G., \& Rodgers, S. (2018). First responder mental healthcare: Evidence-based prevention, postvention, and treatment. Professional Psychology: Research and Practice, 49(3), 193-204. https://doi.org/10.1037/pro0000192

Lee, J.-S., Ahn, Y.-S., Jeong, K.-S. Chae, J.-H., \& Choi, K.-S. (2014). Resilience buffers the impact of traumatic events on the development of PTSD symptoms in firefighters. Journal of Affective Disorders, 162, $128-133$. https://doi.org/10.1016/j.jad.2014.02.031

Lewis, G. B., \& Pathak, R. (2014). The employment of veterans in state and local government service. State and Local Government Review, 46(2), 91-105. https://doi.org/10.1177/0160323X14537835

McCanlies, E. C., Gu, J. K., Andrew, M. E., Burchfiel, C. M., \& Violanti, J. M. (2017). Resilience mediates the relationship between social support and post-traumatic stress symptoms in police officers. Journal of Emergency Management, 15(2), 107-116. https://doi.org/10.5055/jem.2017.0319

National Institute of Mental Health. (2017). Post-traumatic stress disorder. https://www.nimh.nih.gov/health/ statistics/post-traumatic-stress-disorder-ptsd.shtml

Osman, A., Bagge, C. L., Gutierrez, P. M., Konick, L. C., Kopper, B. A., \& Barrios, F. X. (2001). The Suicidal Behaviors Questionnaire-revised (SBQ-R): Validation with clinical and nonclinical samples. Assessment, 8(4), 443-454. https://doi.org/10.1177/107319110100800409

Prosek, E. A., \& Ponder, W. N. (2021). Validation of the Adapted Response to Stressful Experiences Scale (RSES-4) among veterans [Manuscript submitted for publication]. 
Spitzer, R. L., Kroenke, K., Williams, J. B. W., \& Löwe, B. (2006). A brief measure for assessing generalized anxiety disorder (The GAD-7). Archives of Internal Medicine, 166(10), 1092-1097. https://doi.org/10.1001/archinte.166.10.1092

Steinkopf, B., Reddin, R. A., Black, R. A., Van Hasselt, V. B., \& Couwels, J. (2018). Assessment of stress and resiliency in emergency dispatchers. Journal of Police and Criminal Psychology, 33(4), 398-411. https://doi.org /10.1007/s11896-018-9255-3

Substance Abuse and Mental Health Services Administration. (2018, May). First responders: Behavioral health concerns, emergency response, and trauma. Disaster Technical Assistance Center Supplemental Research Bulletin. https://www.samhsa.gov/sites/default/files/dtac/supplementalresearchbulletin-firstrespondersmay2018.pdf

Weiss, D. S., Brunet, A., Best, S. R., Metzler, T. J., Liberman, A., Pole, N., Fagan, J. A., \& Marmar, C. R. (2010). Frequency and severity approaches to indexing exposure to trauma: The Critical Incident History Questionnaire for police officers. Journal of Traumatic Stress, 23(6), 734-743. https://doi.org/10.1002/jts.20576

White, B., Driver, S., \& Warren, A. M. (2010). Resilience and indicators of adjustment during rehabilitation from a spinal cord injury. Rehabilitation Psychology, 55(1), 23-32. https://doi.org/10.1037/a0018451

Wild, J., El-Salahi, S., Degli Esposti, M., \& Thew, G. R. (2020). Evaluating the effectiveness of a group-based resilience intervention versus psychoeducation for emergency responders in England: A randomised controlled trial. PLoS ONE, 15(11), e0241704. https://doi.org/10.1371/journal.pone.0241704

Windle, G., Bennett, K. M., \& Noyes, J. (2011). A methodological review of resilience measurement scales. Health and Quality of Life Outcomes, 9, Article 8, 1-18. https://doi.org/10.1186/1477-7525-9-8 